\title{
Composición de dinoflagelados epífitos y forófitos en la Costa norte del golfo de Morrosquillo, Sucre, Colombia
}

\section{Composition of epiphytic dinoflagellates and phorophytes from north coast of Morrosquillo Gulf, Sucre, Colombia}

Quintana-M Humberto ${ }^{1}$ Biol; Mercado-Gómez Jorge ${ }^{1}$ M.Sc.

${ }^{1}$ Universidad de Sucre, Departamento de Biología y Química, Grupo Evolución y Sistemática tropical, Sincelejo, Colombia.

\section{Keywords:}

Microalgae;

phorophytes;

benthic;

macrophyte;

Caribbean;

Colombia.

\section{Palabras Clave:}

Microalgas;

forófitos;

bentónico;

macrófitas;

Caribe;

Colombia.

\begin{abstract}
To determinate the species composition and specificity of the epiphytic dinoflagellates to phorophytes of the northern coast of the Gulf of Morrosquillo (Sucre-Colombia), were established transects at five points where were collected macrophytes and floating elements for four months. From these, samples of epiphytes dinoflagellates were identified and quantified, in order to establish richness and abundance; In addition, a cluster analysis with Bray-Curtis distance was implemented to establish similarity between the sampling points. Six taxa were found: Prorocentrum lima, $P$. emarginatum, Ostreopsis ovata, O. lenticularis, Cabra cf. aremorica y Coolia sp. Cabra cf. aremorica which is a new record for Colombia and the South Caribbean. Ostreopsis was the most abundant genus, being a rare trend in the Caribbean, where the highest values are provided by Prorocentrum species. Four of the species found are potentially toxic, as they can cause diarrheal intoxication and ciguatera. Assemblages of epiphyte dinoflagellates from the northern coast of the Gulf of Morrosquillo were characterized by a low number of species and the dominance of Ostreopsis species.
\end{abstract}

\section{Resumen}

Con el objetivo de determinar la composición de especies y especificidad de los dinoflagelados epífitos hacia forófitos de la costa norte del Golfo de Morrosquillo (SucreColombia), se establecieron transectos en cinco puntos donde fueron recolectadas macrófitas y elementos flotantes durante cuatro meses. A partir de estas, muestras de dinoflagelados epífitos fueron identificados y cuantificados, con el fin de establecer la riqueza y abundancia; además se implementó un análisis de conglomerados con distancia de Bray-Curtis para establecer similitud entre los puntos de muestreo. Se encontraron seis taxones: Prorocentrum lima, $P$. emarginatum, Ostreopsis ovata, $O$ lenticularis, Cabra cf. aremorica y Coolia sp. Cabra cf. aremorica la cual es un nuevo registro para Colombia y el Caribe sur. El género que mayor abundancia aportó fue Ostreopsis, siendo una tendencia poco común en el Caribe, donde los mayores valores son aportados por especies de Prorocentrum. Cuatro de los taxones encontrados son potencialmente tóxicos (pueden ocasionar intoxicación diarreica y ciguatera). Los ensamblajes de dinoflagelados epífitos de la costa norte del Golfo de Morrosquillo, se caracterizaron por un bajo número de especies y la dominancia de las especies de Ostreopsis. 


\section{Introducción}

Los dinoflagelados son un grupo de microorganismos que forman parte fundamental en las redes tróficas de los ecosistemas marinos, tanto en la zona planctónica como bentónica (TAYLOR et al., 2008; HOPPENRATH et al., 2009). Sin embargo, los estudios sobre su riqueza son pocos y se han enfocado en especies planctónicas, siendo escasos los trabajos sobre taxones bentónicos (AGUILAR-TRUJILLO et al., 2014), tanto de los psamófilos, como de los epífitos (TAYLOR et al., 2008). En el caso de los epífitos, las investigaciones se han enfocado geográficamente en las zonas insulares del Caribe (BESADA et al., 1982; BALLANTINE et al., 1985; BALLANTINE et al., 1988; BOMBER et al., 1988b; BOURDEAU et al., 1995; DELGADO et al., 2006; RODRÍGUEZ et al., 2010; PERAZA y MOREIRA, 2012), incluyendo Colombia (RODRÍGUEZ et al., 2010; MANCERA-PINEDA et al., 2014). En otras palabras, el conocimiento sobre la riqueza de dinoflagelados epífitos en zonas continentales como el Golfo de Morrosquillo (Sucre, Colombia) es desconocida.

Analizar la riqueza de estos microorganismos es de suma importancia, ya que se ha establecido por medio de estudios de composición, que la alta abundancia de algunas especies de los géneros: Amphidinium, Coolia, Gambierdiscus, Ostreopsis, y Prorocentrum están asociados con grandes concentraciones de una amplia variedad de toxinas (DELGADO et al., 2006). Estas toxinas han causado grandes pérdidas económicas a la industria pesquera y turística (ANDERSON y LOBEL, 1987) debido a la contaminación de la fauna íctica consumida por el hombre (ANDERSON y LOBEL, 1987). Asimismo, conocer la composición de especies permitiría la identificación de los taxones que pueden generar compuestos que tienen un fuerte efecto antifúngico (NAGAl et al., 1992), los cuales tienen un potencial uso como fuente alternativa a los fungicidas convencionales usados en los cultivos y en la producción de fármacos (ENCARNAÇÃO et al., 2015).

De igual forma, a nivel taxonómico, este tipo de análisis brinda la posibilidad de establecer nuevos registros para la región y el país (LOZANO-DUQUE et al., 2011), además de sentar base para posteriores trabajos taxonómicos, ecológicos, biotecnológicos y toxicológicos para la región Caribe.

Según lo anterior el presente estudio tuvo como objetivo, determinar la composición de los dinoflagelados epífitos y sus forófitos.

\section{Materiales y Métodos}

Área de estudio: Se encuentra ubicada en la costa norte del Golfo de Morrosquillo, entre las coordenadas

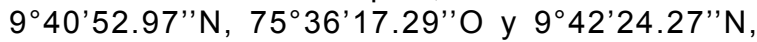
$75^{\circ} 39^{\prime} 31.34^{\prime \prime} \mathrm{O}$, en el municipio de San Onofre, Sucre (Figura 1). Esta zona está formada por colinas bajas de origen denudacional, asociadas al cinturón del Sinú. Sus playas están compuestas por terrazas coralinas y sedimentos formados por arenas litoclásticas y biolitoclásticas de origen terrígeno. Muchas playas se encuentran formadas frente a pantanos de manglar (POSADA POSADA y HENAO PINEDA, 2009).

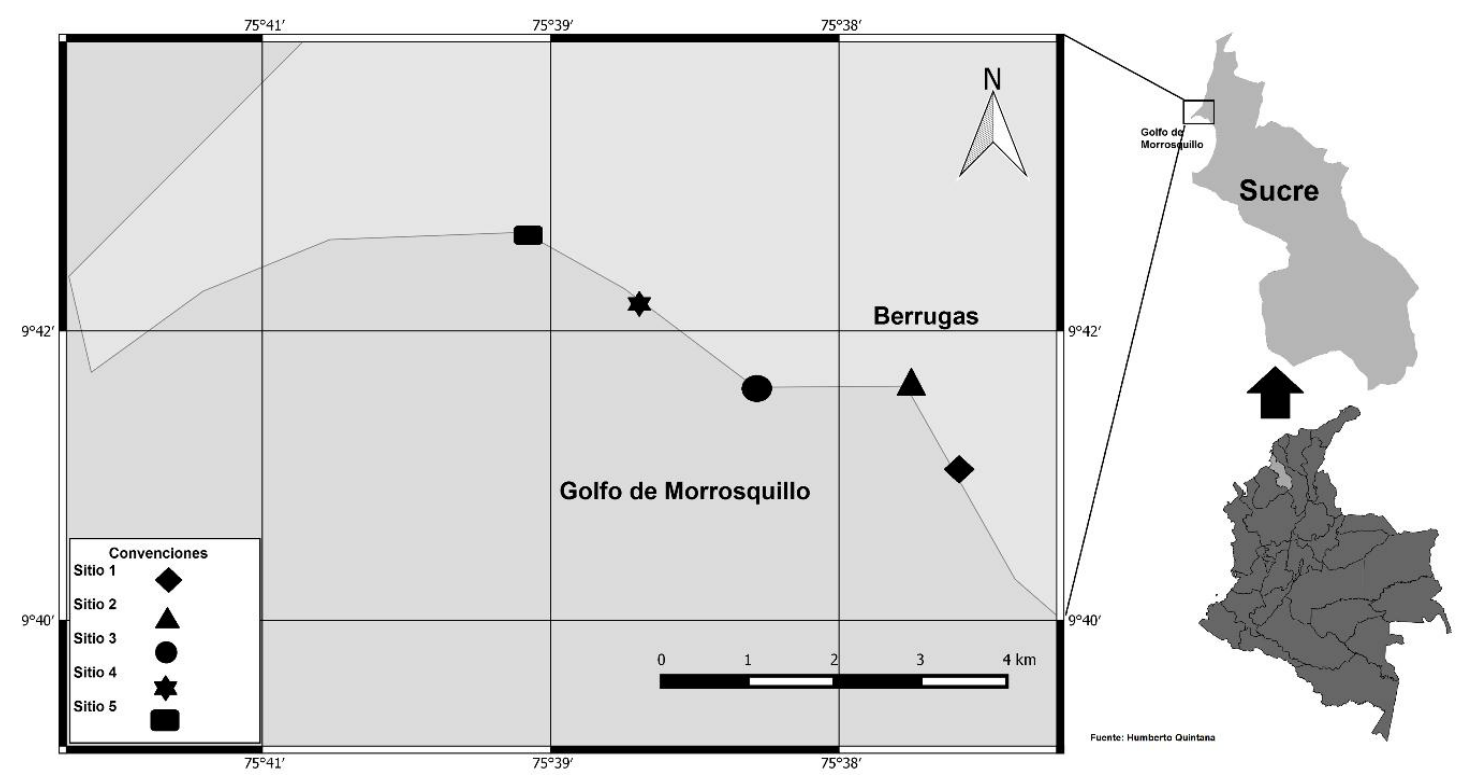

Figura 1. Área de estudio y los puntos de muestreo en la costa norte del Golfo de Morrosquillo, Sucre, Colombia. 
Fase de campo: Se realizaron muestreos mensuales durante un periodo de cuatro meses (febrero - mayo del 2015), en los cuales se establecieron cinco puntos de recolecta (Figura 1). Estos fueron elegidos de acuerdo con la presencia de macrófitas y material orgánico flotante. Entre cada punto de muestreo existió una distancia mínima de un kilómetro, siguiendo lo recomendado por MARGALEF (1978). Igualmente, se tomaron apuntes sobre las características físicas de cada sitio como: presencia de rompeolas, incidencia de actividades humanas, sustrato dominante y coordenadas con un GPS marca GARMIN eTrex 20x.

Sitio 1: Playa arenosa con amplia exposición al oleaje, carente de sustrato rocoso, con excepción de un rompeolas. Presenta poco tránsito de personas. Se encuentra cercana a una zona de pastoreo de ganado. Sitio 2: Playa arenosa con amplia exposición al oleaje, presenta movimiento continuo de lanchas y canoas, gran flujo de personas y descargas de aguas residuales. Sitio 3: Playa arenosa con amplia exposición al oleaje, extensa cobertura de pastos marinos, fragmentos de roca caliza dispersos, producto de la desaparición de un antiguo rompeolas. A pocos metros se halla la desembocadura de un arroyo proveniente de un bosque de manglar.

Sitios 4 y 5: Playas rocosas de origen coralino con amplia exposición al oleaje, extensa cobertura de macroalgas acompañada de pequeños parches de pastos marinos. El sitio cinco se caracteriza por estar cerca de la desembocadura de un arroyo proveniente de un bosque de manglar.

En cada zona de muestreo se efectuó un transecto en banda; este se hizo con una cuerda de $10 \mathrm{~m}$ a partir del borde de la playa que limitó el ancho. Se usó una cuerda con una boya y peso para medir la profundidad, que nunca superó los dos metros. Posteriormente, se hicieron recorridos en zig-zag, con el fin de abarcar la mayor cantidad de individuos de macrófitas posibles (VEGA-SEQUEDA et al., 2008).

Las muestras de macrófitas se tomaron por medio de buceo en apnea y se despegaron con ayuda de una navaja, se almacenaron en recipientes plásticos de 250 o $500 \mathrm{ml}$ dependiendo del tamaño de la macroalga ó angiosperma. Posteriormente, los ejemplares se fijaron en una solución de formol con agua de mar al $4 \%$ y se almacenaron en un recipiente con hielo hasta su análisis en el laboratorio (MITCHELL, 1985; OKOLODKOV et al., 2007; OKOLODKOV et al., 2014). En el caso del material orgánico flotante sólo se tomaron especímenes frescos con el propósito de evitar muestras en mal estado, es decir; evitar ejemplares en descomposición y expuestos por mucho tiempo a radiación solar.

\section{Fase de laboratorio}

Identificación de macrófitas: Inicialmente las muestras fueron separadas en morfotipos de macroalgas, angiospermas y materia orgánica. Posteriormente, se realizaron cortes longitudinales y transversales a mano alzada sobre las estructuras vegetativas y reproductivas. Al interior de cada grupo se utilizaron las claves taxonómicas de TAYLOR (1960), LITTLER y LITTLER (2000), y DAWES y MATHIESON (2008), para la identificación de cada especie. Finalmente, los ejemplares a los cuales no fue posible llegar a una afinidad taxonómica fueron identificados por un experto, el cual también verificaró las especies.

Una vez identificado el material fue procesado según los métodos estándares de herbario (FLOREZ-LEIVA et al., 2010) para: prensado, etiquetado, montaje e inclusión al Herbario de la Universidad de Sucre (HEUS), bajo la enumeración de Quintana H y Mercado- Gómez J.

Identificación de dinoflagelados: Para la identificación de los dinoflagelados, cada muestra fijada fue agitada a mano de forma vigorosa por cinco minutos, con el fin de separar los dinoflagelados de su sustrato. Después, se tomó una alícuota de la muestra y se vertió en una cámara Sedgwick-Rafter para ser observada por medio de un microscopio invertido Nikon Eclipse TS 100. Seguidamente, se aislaron las células con ayuda de una micropipeta y se agregaron en un portaobjetos. Más tarde, se agregó una gota de hipoclorito de sodio al 5 $\%$, para desarticular las placas tecales de las células aisladas (BALECH, 1979) y ser observadas por medio de un microscopio óptico Labomed LX 400.

Para determinar los dinoflagelados, se utilizaron las descripciones y claves taxonómicas de HANSEN et al. (2001), PENNA et al. (2005), MOHAMMAD-NOOR et al. (2005) y AL-YAMANI y SABUROVA (2010).

Abundancia de dinoflagelados epífitos: Inicialmente, las macrófitas fueron secadas con papel absorbente durante un periodo de 72 horas, el cual fue renovado cada cinco horas. Finalizado el proceso de secado, se procedió al pesado en una balanza electrónica OHAUS Scout Pro.

Posteriormente, fueron cuantificados los individuos de cada especie de dinoflagelado, tomando $1 \mathrm{ml} \mathrm{de}$ la alícuota de la muestra, la cual fue vertida, en una cámara de conteo Sedgwick-Rafter. Subsiguientemente, se agregaron una o dos gotas de la solución acuosa de azul tripano al 0.2\% (TAYLOR, 1978), con el fin de teñir las tecas de los dinoflagelados y así, mejorar el proceso de identificación y conteo. 
El valor total de células de diferentes especies se calculó multiplicando el número de células contenido en $1 \mathrm{ml}$, por el volumen total de la muestra (sin previa concentración) y se dividió entre el peso de la macrófita (gramos). Por tal razón, la abundancia de las células es dada en células por gramo de peso húmedo de sustrato (PHS) (BOMBER et al., 1988b; DELGADO et al., 2006; OKOLODKOV et al., 2007).

Análisis de datos: Para observar la similitud entre los diferentes sitios de muestreo se realizó un análisis de conglomerados con distancia de Bray-Curtis. Este análisis fue realizado con el programa PASTPaleontological Statistics versión 3.14 (HAMMER et al., 2001).

\section{Resultados}

Composición de especies de macrófitas: Se registraron un total de 26 especies de macrófitas dividas en 18 géneros, 15 familias y cuatro filos (Tabla 1). La familia Caulerpaceae presentó la mayor riqueza (cinco especies). Las especies de esta familia crecieron principalmente en los sitios uno y dos, donde Caulerpa verticillata y $C$. sertulariodes formaban densos mantos que cubrían las rocas de estos lugares, las cuales estaban cubiertas por una gruesa capa de sedimento. En términos de riqueza, las algas rojas (Rhodophyta) tuvieron 11 especies, seguida de las algas verdes (Chlorophyta) con 7, mientras las algas pardas (Phaeophyta) presentaron cinco especies, y, por último, se encontraron tres especies de pastos marinos.

Composición de especies de dinoflagelados epífitos: Se analizaron en total 245 muestras provenientes de cinco sitios de muestreo de la costa norte del Golfo de Morrosquillo, en las cuales se observaron seis especies, que pertenecen a cuatro géneros de tres familias (Figura 2 y Tabla 2). Los géneros con mayor número de taxones fueron Prorocentrum y Ostreopsis (dos especies); mientras Cabra y Coolia solo una.

El sitio con mayor riqueza fue el sitio tres con seis taxones, mientras que en los otros sitios solo se observaron cuatro (Tabla 2). Todos los sitios fueron dominados principalmente por Prorocentrum y Ostreopsis; los cuales, comprenden más del $80 \%$ de la abundancia total de las células en el área de estudio (Figura 3). La máxima abundancia que presentó cada una de las especies fueron las siguientes: $O$. lenticularis con 67.760 células/g peso húmedo de sustrato (PHS), sobre la macroalga Coelothrix irregularis; mientras $O$. ovata, $P$. lima y Coolia sp. tuvieron concentraciones de $48.800,40.500$ y 24.800 células/g PHS sobre $T$. testudinum, respectivamente. Con una menor cantidad se establecieron $P$. emarginatum con 2.100 células $/ g$ PHS asociado a Syringodium filiforme y Cabra cf. aremorica 1313 células/g PHS sobre Gracilaria sp.
Tabla 1. Especies de macrófitas asociadas a dinoflagelados epífitos de la costa norte del Golfo de Morrosquillo. (*) Nuevos registros para el departamento de Sucre.

\begin{tabular}{|c|c|c|}
\hline Familia & Especies & Sitio \\
\hline \multicolumn{3}{|c|}{ Chlorophyta } \\
\hline Polyphysaceae & Acetabularia calyculus J. V. Lamouroux * & 1 y 3 \\
\hline Bryopsidaceae & Bryopsis pennata J. V. Lamouroux* & 1 \\
\hline \multirow[t]{5}{*}{ Caulerpaceae } & Caulerpa cuppresoides (Vahl) C. Agardh* & 5 \\
\hline & Caulerpa racemosa (Forsskal) J. Agardh & 3 \\
\hline & Caulerpa sertulariodes (S. G. Gmelin) M. Howe & $1,2,3,5$ \\
\hline & Caulerpa verticillata J. Agardh* & $1,2,3$ \\
\hline & Caulerpa taxifolia (M. Vahl) C. Agardh & 3 \\
\hline \multirow{5}{*}{ Dictyotaceae } & $\begin{array}{l}\text { Phaeophyta } \\
\end{array}$ & \\
\hline & $\begin{array}{c}\text { Dictyota menstrualis (Hoyt) Schnetter, Hörning \& } \\
\text { Weber-Peurkert }\end{array}$ & 3,5 \\
\hline & Dictyota pulchella Hörnig \& Schnetter* & 3 \\
\hline & Padina pavonica (Linnaeus) Thivy* & 1 \\
\hline & $\begin{array}{c}\text { Canistrocarpus cervicornis (Kützing) De Paula \& } \\
\text { De Clerck }\end{array}$ & $2,4,5$ \\
\hline \multirow[t]{2}{*}{ Sargassaceae } & Sargassum fluitans (M. Vahl) Børgesen* & 1,3 \\
\hline & Rhodophyta & \\
\hline Rhodomelaceae & Acanthophora spicifera (M.Vahl) Børgesen* & $1,2,3,4,5$ \\
\hline Champiaceae & Coelothrix irregularis (Harvey) Børgesen* & 5 \\
\hline Ceramiaceae & Centroceras micracantum Kützing* & 1,5 \\
\hline Gelidiellaceae & Gelidiella acerosa (Forsskål) Feldmann \& Hamel & 1 \\
\hline Lomentariaceae & Gelidiopsis planicaulis (W.R.Taylor) W.R.Taylor* & 3 \\
\hline \multirow[t]{3}{*}{ Gracilariaceae } & Gracilaria cf. blodgettii Harvey* & 2,5 \\
\hline & $\begin{array}{c}\text { Gracilaria cf. flabelliformis (P. Crouan \& H. } \\
\text { Crouan) Fredericq \& Gurgel }{ }^{*}\end{array}$ & 1,4 \\
\hline & Gracilaria sp. & $2,3,4$ \\
\hline \multirow[t]{2}{*}{ Cystocloniaceae } & Hypnea musciformis (Wulfen) J. V. Lamouroux & 4 \\
\hline & Hypnea spinella (C. Agardh) Kützing* & 4 \\
\hline \multirow[t]{2}{*}{ Soleriaceae } & Soleria filiformis (Kützing) P. W. Gabrielson* & 5 \\
\hline & Magnoliophyta & \\
\hline Cymodoceaceae & Syringodium filiforme Kützing & $1,2,3$ \\
\hline \multirow[t]{2}{*}{ Hidrocharitaceae } & Thalassia testudinum K. D. Koenig & $1,2,3,5$ \\
\hline & Halophila decipiens Ostenfeld & 3 \\
\hline
\end{tabular}
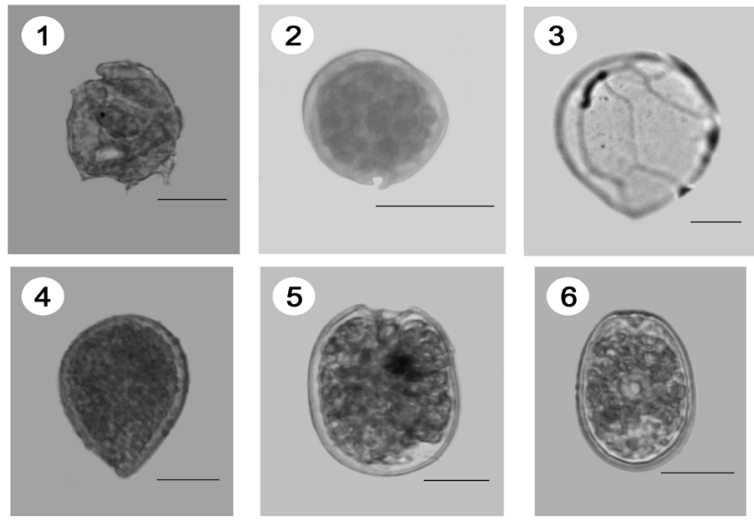

Figura 2. Especies de dinoflagelados epífitos de la costa norte del Golfo de Morrosquillo: (1) Cabra cf. aremorica, (2) Coolia sp., (3) Ostreopsis lenticularis, (4) Ostreopsis ovata, (5) Prorocentrum emarginatum, (6) Prorocentrum lima. Escala $20 \mu \mathrm{m}$. 
Tabla 2. Especies de dinoflagelados epífitos de la costa norte del Golfo de Morrosquillo y los sitios donde fueron observados.

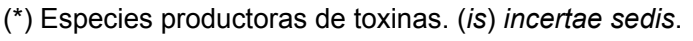

\begin{tabular}{ccc}
\hline Familia & Especie & Sitio \\
\hline \multirow{2}{*}{ Prorocentraceae } & Prorocentrum lima* (Ehrenberg) F. Stein & $1,2,3,4,5$ \\
& $\begin{array}{c}\text { Prorocentrum emarginatum }{ }^{*} \text { Y. Fukuyo } \\
\text { Ostreopsis ovata D. R. Norris, J. W Bomber \& }\end{array}$ & 3,5 \\
Balech & $1,2,3,4,5$ \\
Goniodomaceae & Ostreopsis lenticularis* Y. Fukuyo & $1,2,3,4,5$ \\
& Coolia sp* & $1,2,3,4,5$ \\
Peridiniaceae (is) & Cabra cf. aremorica Chomérat, Couté \& Nézan & 3
\end{tabular}

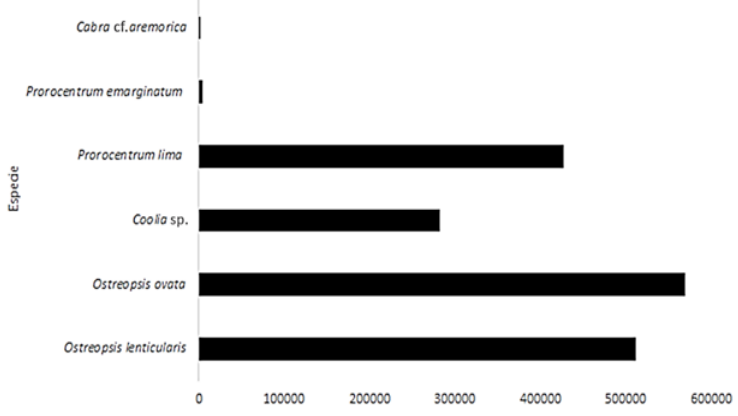

Abundancia células $g-1$ peso húmedo

Figura 3. Abundancia total de dinoflagelados epífitos de la costa norte del Golfo de Morrosquillo.

El sitio con mayor abundancia fue el tres con un valor total de 37.0664 células/g PHS (Figura 4). En contraste, los sitios con menor abundancia fueron los sitios uno y dos (100406 y 144263 células/g PHS en total, respectivamente), con excepción de Coolia sp., que presentó una abundancia total de 21.600 células/g PHS sobre Briopsis pennata (sitio uno). También en estos dos sitios se presentaron el mayor número de especies de macrófitas sin dinoflagelados asociados.

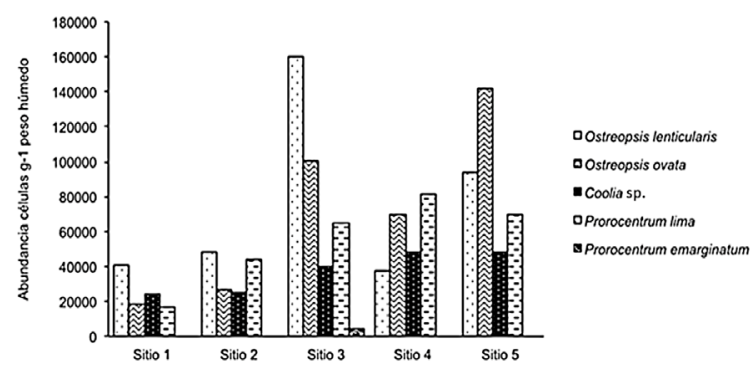

Figura 4. Abundancia total de dinoflagelados epífitos por sitio en la costa norte del Golfo .de Morrosquillo.

Similitud entre sitios de muestreo: A través del análisis de similitud (Figura 5) se pudo observar la formación de dos grandes grupos. El primero, conformado por los sitios tres, cuatro y cinco, los cuales soportaron las mayores abundancias y riqueza de especies, con una semejanza de más del $75 \%$. La segunda agrupación formada por los sitios uno y dos, los cuales tuvieron los valores más bajos de abundancia y riqueza.

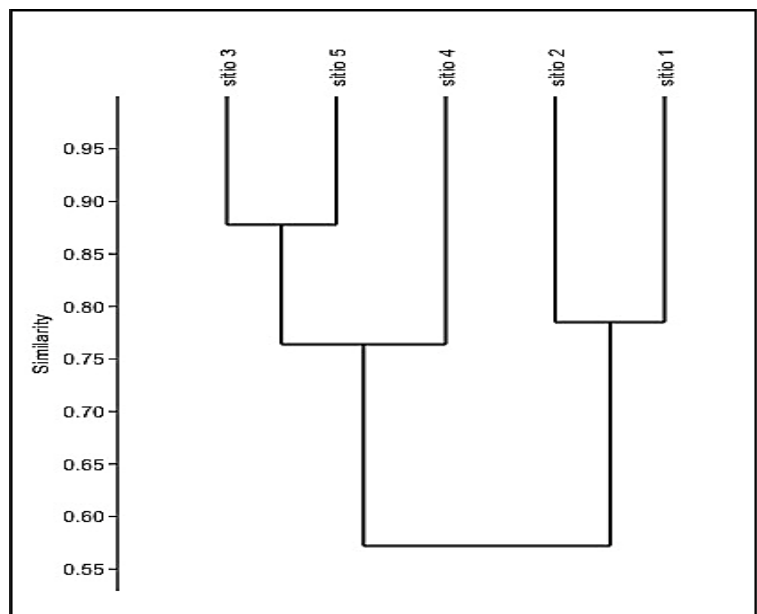

Figura 5. Agrupamiento de similitud de Bray Curtis por sitios de muestreo.

\section{Discusión}

Riqueza de macrófitas: Quince especies de macroalgas son nuevos registros para el departamento de Sucre, tres algas pardas, cuatro verdes y ocho rojas (Tabla 1), incrementando el número de taxones registrados para el departamento. La composición de las especies de macroalgas de la costa norte del gofo de Morrosquillo es típica de aguas tropicales, en las cuales las algas rojas son el grupo más rico en especies (MATEO-CID et al., 2013).

Con respecto a la presencia de especies en los sitos, el pasto marino $T$. testudinum estuvo distribuido en todos los sitios, aunque solamente dominó en el tres, en los otros lugares solo fue hallado formando pequeños parches no mayores de $5 \mathrm{~m}^{2}$. Esta especie se encontró en estrecha relación con $S$. filiforme, la cual se distribuyó en las partes más someras de la costa. Asimismo, el sitio tres presentó el mayor número de especies de macrófitas, debido a la presencia de una amplia cobertura de sustrato duro que favorece el desarrollo de macroalgas (MATEO-CID et al., 2013). Asimismo, la mayoría de los sitios estuvieron conformados por macroalgas pertenecientes a los géneros Caulerpa, Padina y Dictyota. En ciertos casos, algunas de las especies de estos géneros formaron praderas monoespecíficas.

Las muestras de material orgánico flotante fueron escasas, solamente se encontraron cuatro unidades de 245 muestras de macrófitas analizadas en total. De las cuatro, tres pertenecieron a Sargassum fluitans, mientras que la última muestra fue un fragmento de madera. Esto se pudo deber a que el muestreo se desarrolló en un periodo seco, el cual carece de 
fuerte oleaje, lluvias y tormentas que favorecen el desprendimiento y movimiento de material en las costas.

El número de especies de macrófitas asociadas a dinoflagelados epífitos encontradas en este estudio, es uno de los más altos del Caribe, después de Yucatán con 37 (OKOLODKOV et al., 2014). Para Colombia, hasta el momento este es el mayor número de taxones de macrófitas asociadas a dinoflagelados epífitos (Tabla 2), seguido de la isla de San Andrés con 12 (RODRÍGUEZ et al., 2010).

Composición y abundancia de los dinoflagelados epífitos: La riqueza de especies de dinoflagelados epífitos en la costa norte del Golfo del Morrosquillo es baja en comparación con otros sitios del Gran Caribe. Como el Sistema Arrecifal Veracruzano en el suroeste del Golfo de México (OKOLODKOV et al., 2007) donde fueron encontradas 16 taxones, al igual que en la costa norte de Yucatán con 26 (AGUILAR-TRUJILLO et al., 2014; OKOLODKOV et al., 2014) y la parte norte del Sistema Arrecifal Mesoamericano con 24 (ALMAZÁNBECERRIL et al., 2015). El bajo número de taxones encontrados, puede ser consecuencia de dos factores: a) la metodología empleada para el muestreo y fijación. Esta excluye células atecadas o débilmente tecadas, las cuales fueron reportadas en estudios mencionadas anteriormente. La cuantificación e identificación de estos microorganismos requieren de protocolos, técnicas y equipos más específicos de difícil acceso (ESCOBAR-MORALES y HERNÁNDEZ-BECERRIL, 2015). b) El tiempo de muestreo. En su mayoría las investigaciones realizadas en este campo comprenden períodos más extensos (durante diferentes estaciones climáticas o por un año) ya que la dinámica de las poblaciones de estos microorganismos es impredecible (ALMAZÁN-BECERRIL et al., 2015). Empero, que los muestreos fueran propuestos en un diseño que contemplaran periodos de sequía (febrero y marzo) y lluvia (abril y mayo) no fue suficiente para abarcar la diversidad de estos organismos, ya que durante el año de muestreo, se presentó uno de los períodos de sequía más graves de los últimos 10 años (CHAN et al., 2015). Lo anterior no permitió que se desarrollaran lluvias en el área de muestreo durante el periodo de estudio, de tal forma que no fue posible evaluar el comportamiento poblacional de estas especies en temporada de lluvia.

Por otra parte, los trabajos sobre dinoflagelados se han enfocado principalmente en arrecifes de coral (YASUMOTO et al., 1979; KOHLER y KOHLER, 1992; MORTON y FAUST, 1997; OKOLODKOV et al., 2007). Esto se dio a causa de los múltiples reportes de intoxicaciones con ciguatera estuvieron asociados con peces extraídos de arrecifes en el pacífico (YASUMOTO et al., 1979) haciendo de estos ambientes un punto de estudio en la búsqueda de especies productoras de toxinas en todo el mundo; sin embargo, los resultados de este y otros estudios como los de VALERIO y DÍAZ (2008), RODRÍGUEZ et al. (2010), MARTÍNEZ-CRUZ et al. (2015) y NAVARRO-VARGAS et al. (2014) muestran que las praderas de pastos marinos son un hábitat que pueden soportar especies de dinoflagelados epífitos, algunos de ellos tóxicos.

Con respecto a la composición, los géneros encontrados en este estudio son típicos dentro de los ensamblajes de dinoflagelados epífitos del Caribe, con excepción del género Cabra (Fig. 2 y Tabla 2). (ALMAZÁN-BECERRIL et al., 2015; DELGADO et al., 2006; OKOLODKOV et al., 2014). No obstante, es posible que la riqueza sea mayor, ya que en el microfitobentos del Caribe también han sido encontradas especies de los géneros Gambierdiscus, Amphidinium, Plagiodinium, Bysmatrum y Sinophysis (ALMAZÁN-BECERRIL et al., 2015; OKOLODKOV et al., 2014). La carencia de estos grupos pudo ser el resultado de la gran abundancia de Ostreopsis o de la fuerte sequía presentada en el Caribe (producto del fenómeno del niño mencionado anteriormente). Con respecto a la presencia de Ostreopsis, algunos estudios han demostrado que el número de células de Gambierdiscus disminuyen y se incrementan las de Ostreopsis, lo cual puede ser reflejo de desplazamiento por competencia o alelopatía (RICHLEN y LOBEL, 2011).

Según lo anterior, Ostreopsis fue el género más frecuente y abundante durante el estudio. Aportando la mayor abundancia con más del $50 \%$ total de células (1080621 células/g PHS) (Figura 3), una tendencia poco común dentro de los estudios en el Caribe, donde $P$. lima (BOMBER et al., 1988a; BOMBER et al., 1989; DELGADO et al., 2006; MARTÍNEZ-CRUZ et al., 2015) y $P$. mexicanum (AGUILAR-TRUJILLO et al., 2014; OKOLODKOV et al., 2014) normalmente aportan las abundancias más altas. Por lo tanto, los ensamblajes de dinoflagelados de la costa norte del golfo de Morrosquillo, son extrañamente más a fines con los encontrados en aguas del Mar Mediterráneo o costas con clima templado (RHODES et al., 2000; VILA et al., 2001; SHEARS y ROSS, 2009; COHU y LEMÉE, 2012) que con ambientes tropicales.

A nivel de especies, $P$. lima es un taxón que presenta una distribución geográfica muy amplia y se puede encontrar tanto en aguas templadas como cálidas (NAGAHAMA et al., 2011; GLIBERT et al., 2012). En este estudio, la máxima abundancia mostrada por $P$. lima fue de 40500 células/g PHS sobre T. testudinum, este valor es menor al reportado en Cartagena como un florecimiento algal nocivo (FAN) 4,5 × $10^{6}$ células $\mathrm{L}^{-1}$ (SALON-BARROS et al., 2016). En este contexto, es importante puntualizar que en el Golfo de Morrosquillo 
esta especie puede incrementar sus valores y ser completamente nociva para el hombre; por lo tanto, un seguimiento a sus poblaciones es de gran relevancia para la región.

Asimismo, $P$. emarginatum también es una especie ampliamente distribuida, pero reportada con un bajo número de células en aguas tropicales y subtropicales con registros en el océano Pacífico (OKOLODKOV y GÁRATE-LIZÁRRAGA, 2006; MURRAY et al., 2007), Índico (GRZEBYK et al., 1998), mar Caribe (RODRÍGUEZ et al., 2010; MANCERA-PINEDA et al., 2014) y el Mediterráneo (ALIGIZAKI et al., 2009; LAZA-MARTINEZ et al., 2011). En el área de muestreo presentó la densidad más alta reportada hasta el momento en el país con 2100 células/g PHS asociadas a S. filiforme, superando por un estrecho margen a San Andrés con 1980 células/g PHS (MANCERA-PINEDA et al., 2014). No obstante, todavía se encuentra dentro de los márgenes establecidos en otras localidades (ALIGIZAKI et al., 2009; SHAH et al., 2013). Estos resultados permiten sugerir que este linaje siempre presenta poblaciones con un bajo número de células en diferentes regiones del mundo, incluyendo costas colombianas.

El género Coolia está conformado por siete especies (GUIRY y GUIRY, 2017); sin embargo en la zona de estudio solo fue hallada un morfotipo al cual no fue posible relacionarlo con ningún epíteto específico, lo cual es producto de dos factores: a) las dificultades taxonómicas reportadas en la identificación de estas especies (KARAFAS et al., 2015), ó b) la asociación a una nueva entidad taxonómica, teniendo en cuenta que en años recientes varios taxones han sido descritos como nuevos en todo el mundo (ALMAZÁN-BECERRIL et al., 2015).

Varias especies de Coolia están asociadas a la producción de ictiotoxinas y citotoxinas (HOLMES et al., 1995; RHODES y TOMAS, 1997), por lo tanto es requerido un estudio más profundo de estas células para mejorar su identificación y así establecer si las poblaciones del Golfo de Morrosquillo son potencialmente tóxicas, teniendo en cuenta su alta abundancia ( 24800 células/g PHS); estas enormes concentraciones en aguas tropicales son datos atípicos, ya que el mayor valor de 3005 células/g PHS solo ha sido reportado en el Sistema Arrecifal Veracruzano en el suroeste de México (OKOLODKOV et al., 2007). La alta abundancia registrada pudo deberse a la turbulencia modera y altas temperaturas que se registraron en el período de muestreo (RHODES et al., 2000).
El género Cabra es un nuevo registro para el Caribe sur y Colombia. Solamente se halló una especie, la cual se le atribuyó a Cabra cf. aremorica. La identificación de este individuo no es $100 \%$ verificable usando únicamente los caracteres a través de un microscopio de luz; ya que presenta una gran similitud hacia $C$. reticulata (GÓMEZ y LOPES, 2015). Hasta el momento, este género había sido reportado en dos países del Caribe (México y Puerto Rico) asociados a praderas de pastos marinos (OKOLODKOV et al., 2007; OKOLODKOV et al., 2014; GÓMEZ y LOPES, 2015), indicando lo extraña y escasa que pueden ser las especies de este grupo. Además, de los taxones anteriormente mencionados, con excepción de Cabra cf. aremorica, todas tienen la capacidad de producir toxinas, que pueden contaminar la fauna piscícola (DELGADO et al., 2006).

Cabe resaltar que solamente en 24 de las 245 muestras de forófitos analizadas, no se encontraron dinoflagelados, lo que indica que estos microorganismos pueden ser encontrados sobre una gran variedad de forófitos independientemente de la especie de sustrato (DELGADO et al., 2006).

Por último, las muestras de material orgánico flotante soportaron una baja abundancia de células, pues el valor máximo fue de 870 células/g PHS. Esto se pudo deber la fuerte incidencia de luz que se encuentra sobre este tipo de material, siendo un factor que limita el crecimiento de estos microorganismos (BOMBER et al., 1988b). BOMBER et al. (1988b) y MANCERA-PINEDA et al. (2014) han demostrado que los ensamblajes de dinoflagelados sobre el material flotante puede jugar un papel muy importante en la dispersión, pues sirven como medio de transporte, cumpliendo papel fundamental sobre la ecología de este grupo.

Similitud entre sitios de muestreo: Los sitios uno y dos mostraron las abundancias más bajas de dinoflagelados epífitos a lo largo del estudio. Una de las posibles causas que pudieron afectar son las características de los sitios, ya que estos están compuestos principalmente por rompeolas, los cuales cumplen la función de disminuir la fuerza de llegada del oleaje a la costa. No obstante, estos ocasionan que incremente la turbulencia dado el choque de estas corrientes hacia las rocas, de tal forma que es posible que la adhesión efectiva de los dinoflagelados epífitos sobre las macrófitas disminuya, haciendo que se suspendan en la columna de agua (NAKAHARA et al., 1996; RICHLEN y LOBEL, 2011). Además, se observó una fuerte deposición de sedimento sobre la mayoría de macrófitas, que también afecta la colonización de estos microorganismos (DELGADO et al., 2006). 
Los sitos tres y cinco mostraron una fuerte relación, esto posiblemente se dio por las siguientes razones. a) Estos lugares se encuentran cerca desembocaduras de pequeños arroyos provenientes de bosques de manglar, los cuales, pueden suplir de nutrientes a las poblaciones de dinoflagelados. b) Las grandes poblaciones de macrófitas que tienen estos dos sitios, implica más sustrato disponible para su colonización. c) Esta zona se encuentra expuesta a turbulencia moderada, en la cual se ha comprobado que favorece el crecimiento de especies del género Ostreopsis las cuales fueron las más abundantes en estos dos sitios (VILA et al., 2001; SHEARS y ROSS, 2009; RICHLEN y LOBEL, 2011; SELINA et al., 2014) .

\section{Conclusiones}

A pesar del bajo número de especies observado, los resultados de este estudio son de gran importancia, ya que reportan por primera vez al género Cabra para el Caribe sur, mientras que todas las especies encontradas son nuevos registros para el departamento de Sucre.
Igualmente, se registran cuatro especies potencialmente toxicas, dado que producen toxinas asociadas a síndromes como la ciguatera y la intoxicación diarreica por consumo de mariscos, siendo una amenaza latente para la salud pública de la zona.

El gran número de taxones de macrófitas encontradas en el corto periodo de estudio indica que la diversidad de este grupo de organismos debe ser mayor en la zona, por lo tanto, se sugiere incrementar el área de muestreo en relación a estudios de diversidad de algas, de tal forma que se pueda establecer una aproximación más real a la diversidad de estos grupos.

Agradecimientos: Al laboratorio de biomédicas de la Universidad de Sucre por su apoyo en el desarrollo de este trabajo, al doctor Yuri Okolodkov del Instituto de Ciencias Marinas y Pesquerías (ICIMAP) por sus comentarios la manuscrito e identificación de dinoflagelados. Del mismo modo, a Brigitte Gavio de la Universidad Nacional por su gran ayuda en la identificación y corroboración de las especies de macroalgas.

\section{Referencias}

AGUILAR-TRUJILLO, A.; OKOLODKOV, Y.; MERINO-VIRGILIO, F.; OSORIO-MORENO, I.; HERRERA-SILVEIRA, J. 2014. Variación espacial de dinoflagelados bentónicos/epifíticos en aguas costeras del norte de Yucatán (agosto de 2011). Págs. 147-160 en: Botello, A.; Rendón von Osten, J.; Benítez, J.; Gold-Bouchot, G. (eds.), Golfo de México. Contaminación e impacto ambiental: diagnóstico y tendencias. Cinvestav-Unidad Mérida: UAC, UNAM-ICMYL.

AL-YAMANI, F.; SABUROVA, M. 2010. Illustrated Guide on the Flagellates of Kuwait's Intertidal Soft Sediments. Kuwait Institute for Scientific Research, Safat, Kuwait.

ALIGIZAKI, K.; NIKOLAIDIS, G.; KATIKOU, P.; BAXEVANIS, A.; ABATZOPOULOS, T. 2009. Potentially toxic epiphytic Prorocentrum (Dinophyceae) species in Greek coastal waters. Harmful Algae 8 (2): 299-311.

ALMAZÁN-BECERRIL, A., ESCOBAR-MORALES, S., ROSILES-GONZÁLEZ, G., VALADEZ, F. 2015. Benthicepiphytic dinoflagellates from the northern portion of the Mesoamerican Reef System. Botanica Marina 58 (2): 115-128.

ANDERSON, D.; LOBEL, P. 1987. The continuing enigma of ciguatera. The Biological Bulletin 172 (1): 89-107.

BALECH, E. 1979. Tres dinoflagelados nuevos o interesantes de aguas brasileñas. Boletín del Instituto de Oceanografía de São Paulo 28 (2): 55-64.

BALLANTINE, D.; BARDALES, A.; TOSTESON, T.; DURST, H. 1985. Seasonal abundance of Gambierdiscus toxicus and Ostreopsis sp. in coastal waters of southwest Puerto Rico. Págs 417-422 en: Gabrie, C.; Marmelin, V. (Eds.), Proceedings of the Fifth International Coral Reef Congress (Vol. 4). Tahiti: Antenne Museum-EPHE.

BALLANTINE, D.; TOSTESON, T.; BARDALES, A. 1988. Population dynamics and toxicity of natural populations of benthic dinoflagellates in southwestern Puerto Rico. Journal of Experimental Marine Biology and Ecology 119 (3): 201-212.

BESADA, E.; LOEBLICH, L.; LOEBLICH III, A. (1982). Observations on tropical, benthic dinoflagellates from ciguateraendemic areas: Coolia, Gambierdiscus, and Ostreopsis. Bulletin of Marine Science 32 (3): 723-735. 
BOMBER, J.; GUILLARD, R.; NELSON, W. 1988a. Roles of temperature, salinity, and light in seasonality, growth, and toxicity of ciguatera-causing Gambierdiscus toxicus Adachi et Fukuyo (Dinophyceae). Journal of Experimental Marine Biology and Ecology 115(1):53-65.

BOMBER, J.; MORTON, S.; BABINCHAK, J.; NORRIS, D.; MORTON, J. 1988b. Epiphytic dinoflagellates of drift algae-another toxigenic community in the ciguatera food chain. Bulletin of Marine Science 43(2):204-214.

BOMBER, J.; NORRIS, D.; MITCHELL, L.; ANDERSON, D. 1985. Benthic dinoflagellates associated with ciguatera from the Florida Keys. II. Temporal, spatial and substrate heterogeneity of Prorocentrum lima. Págs 45-50. en Anderson, D.; White, A.; Banden, D. (eds.), Toxic Dinoflagellates New York.

BOMBER, J.; RUBIO, M.; NORRIS, D. 1989. Epiphytism of dinoflagellates associated with the disease ciguatera: substrate specificity and nutrition. Phycologia 28(3):360-368.

BOURDEAU, P.; DURAND-CLEMENT, M.; AMMAR, M.; FESSARD, V. 1995. Ecological and toxicological characteristics of benthic dinoflagellates in a ciguateric area (Saint Barthelemy: French West Indies). Págs 133-137 en: Lassus, P.; Arzul, G.; Erard, E.; Gentien, P.; Marcou, C. (Eds.), Harmful Marine Algal Blooms. London.

CHAN, S.; KENDON, E.; ROBERTS, N.; FOWLER, H.; BLENKINSOP, S. 2016. Downturn in scaling of UK extreme rainfall with temperature for future hottest days. Nature Geoscience 9 (1): 24-28.

COHU, S.; LEMÉE, R. (2012). Vertical distribution of the toxic epibenthic dinoflagellates Ostreopsis cf. ovata, Prorocentrum lima and Coolia monotis in the NW Mediterranean Sea. CBM-Cahiers de Biologie Marine 53 (3): 373-380.

DAWES, C.; MATHIESON, A. 2008. The seaweeds of Florida. University Press of Florida, Gainesville.

DELGADO, G.; LECHUGA-DEVÉZE, C.; POPOWSKI, G.; TROCCOLI, L.; SALINAS, C. 2006. Epiphytic dinoflagellates associated with ciguatera in the northwestern coast of Cuba. Revista de Biología Tropical 54 (2): 299-310.

ENCARNAÇÃO, T.; PAIS, A.; CAMPOS, M.; BURROWS, H. 2015. Cyanobacteria and microalgae: A renewable source of bioactive compounds and other chemicals. Science progress 98 (2): 145-168.

ESCOBAR-MORALES, S.; HERNÁNDEZ-BECERRIL, D. 2015. Free-living marine planktonic unarmoured dinoflagellates from the Gulf of Mexico and the Mexican Pacific. Botanica Marina 58 (1): 9-22.

FLOREZ-LEIVA, L.; GAVIO, B.; DÍAZ-RUIZ, M.; CAMACHO, O.; DÍAZ-PULIDO, G. 2010. Recolección y preservación de macroalgas marinas: Una guía para estudios ficológicos. INTROPICA 5 (1): 97-103.

GLIBERT, P.; BURKHOLDER, J.; KANA, T. 2012. Recent insights about relationships between nutrient availability, forms, and stoichiometry, and the distribution, ecophysiology, and food web effects of pelagic and benthic Prorocentrum species. Harmful Algae 14: 231-259.

GÓMEZ, F.; LOPES, R. 2015. New records of the distinctive benthic dinoflagellate genus Cabra (Dinophyceae). CICIMAR Oceánides 30 (1): 63-70.

GRZEBYK, D.; SAKO, Y.; BERLAND, B. 1998. Phylogenetic analysis of nine species of Prorocentrum (Dinophyceae) inferred from $18 S$ ribosomal DNA sequences, morphological comparisons, and description of Prorocentrum panamensis, sp. nov. Journal of Phycology 34 (6): 1055-1068.

GUIRY, M.; GUIRY, G. 2017. AlgaeBase. World-wide electronic publication, National University of Ireland, Galway. http://www.algaebase.org.

HAMMER, R.; HARPER, D.; RYAN, P. 2001. PAST: Paleontological statistics software package for education and data analysis. Palaeontologia Electronica 4 (1): 9 pp. http://palaeo-electronica.org/2001_1/past/issue1_01.htm 
HANSEN, G.; TURQUET, J.; QUOD, J.; TEN-HAGE, L.; LUGOMELA, C.; KYEWALYANGA, M.; HURBUNGS, M.; WAWIYE, P.; OGONGO, B.; TUNJE, S.; RAKOTOARINJANAHARY, H. 2001. Potentially harmful microalgae of the Western Indian Ocean: a guide based on a preliminary survey en: IOC Manuales y guías, 41. Comision Oceanográfica Interguvernamental de UNESCO, Paris.

HOLMES, M.; LEWIS, R.; JONES, A.; WONG, A. 1995. Cooliatoxin, the first toxin from Coolia monotis (Dinophyceae). Natural toxins 3 (5): 355-362.

HOPPENRATH, M.; ELBRÄCHTER, M.; DREBES, G. 2009. Marine phytoplankton: Selected microphytoplankton species from the North Sea around Helgoland and Sylt. Schweizerbart'sche Verlag. Sttutgart.

KARAFAS, S.; ROBERT, Y.; CARMELO, T. 2015. Morphological and genetic analysis of the Coolia monotis species complex with the introduction of two new species, Coolia santacroce sp. nov. and Coolia palmyrensis sp. nov. (Dinophyceae) Harmful Algae 46: 18-33.

KOHLER, S.; KOHLER, C. 1992. Dead bleached coral provides new surfaces for dinoflagellates implicated in ciguatera fish poisonings. Environmental Biology of Fishes 35 (4): 413-416.

LAZA-MARTINEZ, A.; ORIVE, E.; MIGUEL, I. 2011. Morphological and genetic characterization of benthic dinoflagellates of the genera Coolia, Ostreopsis and Prorocentrum from the south-eastern Bay of Biscay. European Journal of Phycology 46 (1): 45-65.

LITTLER, D.; LITTLER, M. 2000. Caribbean reef plants. An identification guide to the reef plants of the Caribbean, Bahamas, Florida and Gulf of Mexico. Offshore Graphics. Inc. Washington, DC, 542.

LOZANO-DUQUE, Y.; ALFONSO VIDAL, L.; NAVAS, S.; GABRIEL, R. 2011. Lista de especies de dinoflagelados (Dinophyta) registrados en el mar Caribe colombiano. Boletín de Investigaciones Marinas y Costeras-INVEMAR 40 (2): 361-380.

MANCERA-PINEDA, J.; MONTALVO-TALAIGUA, M.; GAVIO, B. 2014. Dinoflagelados potencialmente tóxicos asociados a material orgánico flotante (DRIFT) en San Andrés Isla, Reserva Internacional de la Biosfera - Seaflower. Caldasia 36 (1): 139-156.

MARGALEF, R. 1978. Sampling Design: Some examples. En A. Sournia (Ed.), Phytoplankton manual. Vol. 6, Págs 17-31. París: Monographs Oceanographic Methodology.

MARTÍNEZ-CRUZ, M.; OKOLODKOV, Y.; AGUILAR-TRUJILLO, A.; HERRERA-SILVEIRA, J. 2015. Epiphytic dinoflagellates on the seagrass Thalassia testudinum at Dzilam, southeastern Gulf of Mexico. Cymbella 1 (3): 1-9.

MATEO-CID, L.; MENDOZA-GONZÁLEZ, A.; ÁVILA-ORTIZ, A.; DÍAZ MARTÍNEZ, S. 2013. Algas marinas bentónicas del litoral de Campeche, México. Acta Botanica Mexicana 104: 53-92.

MITCHELL, L. 1985. Ecological studies of benthic dinoflagellates associated with ciguatera in the Florida Keys: the 0-38 micrometer size fraction. (Tesis de maestría), Florida Institute of Technology, Florida.

MOHAMMAD-NOOR, N.; DAUGBJERG, N.; MOESTRUP, O.; ANTON, A. 2005. Marine epibenthic dinoflagellates from Malaysia--A study of live cultures and preserved samples based on light and scanning electron microscopy. Nordic Journal of Botany 24 (6): 629-690.

MORTON, S.; FAUST, M. 1997. Survey of toxic epiphytic dinoflagellates from the Belizean barrier reef ecosystem. Bulletin of Marine Science 61 (3): 899-906.

MURRAY, S.; NAGAHAMA, Y.; FUKUYO, Y. 2007. Phylogenetic study of benthic, spine-bearing prorocentroinds including prorocentrum fukoyoi sp. nov. Phycological Research 55: 91-102.

NAGAHAMA, Y.; MURRAY, S.; TOMARU, A.; FUKUYO, Y. 2011. Species boundaries in the toxic dinoflagellate Prorocentrum lima (Dinophyceae, Prorocentrales), based on morphological and phylogenetic characters. Journal of Phycology 47 (1): 178-189. 
NAGAI, H.; MURATA, M.; TORIGOE, K.; SATAKE, M.; YASUMOTO, T. 1992. Gambieric acids, new potent antifungal substances with unprecedented polyether structures from a marine dinoflagellate Gambierdiscus toxicus. The Journal of Organic Chemistry 57 (20): 5448-5453.

NAKAHARA, H.; SAKAMI, T.; CHINAIN, M.; ISHIDA, Y. 1996. The role of macroalgae in the epiphytism of de toxic dinoflagellate Gambierdiscus toxicus (Dinophyceae). Phycological Research 44: 113-117.

NAVARRO-VARGAS, G.; DÍAZ-RAMOS, J.; TROCCOLI-GHINAGLIA, L.; SUBERO-PINO, S. 2014. Dinoflagelados epibentónicos presentes en diferentes sustratos en la Bahía Turpialito, golfo de Cariaco, Venezuela. Boletín del Instituto de Oceanografía de Venezuela 53 (2): 161-170.

OKOLODKOV, Y.; CAMPOS-BAUTISTA, G.; GÁRATE-LIZÁRRAGA, I.; GONZÁLEZ-GONZÁLEZ, J.; HOPPENRATH, M.; ARENAS, V. 2007. Seasonal changes of benthic and epiphytic dinoflagellates in the Veracruz reef zone, Gulf of Mexico. Aquatic Microbial Ecology 47 (3): 223-237.

OKOLODKOV, Y.; GÁRATE-LIZÁRRAGA. 2006. An annotated checklist of dinoflagellates (Dinophyceae) from Mexican Pacific. Acta Botánica Mexicana. 74: 1-154.

OKOLODKOV, Y.; MERINO-VIRGILIO, F.; AKÉ-CASTILLO, J.; AGUILAR-TRUJILLO, A.; ESPINOSA-MATÍAS, S.; HERRERA-SILVEIRA, A. 2014. Seasonal changes in epiphytic dinoflagellate assemblages near the northern coast of the Yucatan Peninsula, Gulf of Mexico. Acta Botanica Mexicana 107: 121-151.

PENNA, A.; VILA, M.; FRAGA, S.; GIACOBBE, M.; ANDREONI, F.; RIOBÓ, P.; VERNESI, C. 2005. Characterization of Ostreopsis and Coolia (Dinophyceae) isolates in the Western Mediterranean sea based on morphology, toxicity and Internal Transcribed Spacer 5.8S rDNA sequences. Journal of Phycology 41 (1): 212-225.

PERAZA, R.; MOREIRA, A. 2012. Composición y abundancia de dinoflagelados epibentónicos tecados potencialmente tóxicos de la Ensenada de Guajimico, región centro-sur de Cuba. Revista Cubana de Investigaciones Pesqueras 29 (1): 59-66.

POSADA B.; HENAO, P. 2009. Diagnóstico de la erosión costera del departamento de Sucre, Caribe colombiano. instituto de investigaciones Marinas y costeras "José Benito Vives De Andréis “ (INVEMAR). Santa Marta, Colombia.

RHODES, L.; ADAMSON, J.; SUZUKI, T.; BRIGGS, L.; GARTHWAITE, I. 2000. Toxic marine epiphytic dinoflagellates, Ostreopsis siamensis and Coolia monotis (Dinophyceae), in New Zealand. New Zealand Journal of Marine and Freshwater Research 34 (2): 371-383.

RHODES, L.; THOMAS, A. 1997. Coolia monitis (Dinophyceae) : a toxic epiphytic microalgal species found in New Zeland (note). New Zealand Journal of Marine and Freshwaters Research 31: 139-141.

RICHLEN, M.; LOBEL, P. 2011. Effects of depth, habitat, and water motion on the abundance and distribution of ciguatera dinoflagellates at Johnston Atoll, Pacific Ocean. Marine Ecology Progress Series 421: 51-66.

RODRÍGUEZ, A.; MANCERA-PINEDA, J.; GAVIO, B. 2010. Preliminary Survey of Benthic Dinoflagellates Associated to Beds of Thalassia testudinum in San Andrés Island, Seaflower Biosphere Reserve, Caribbean Colombia. Acta Biológica Colombiana 15 (2): 231-248.

SALON-BARROS, J.; ARREGOCES, R.; MANCERA-PINEDA, H. 2016. Prorocentrum lima and Prorocentrum balticum blooms in Cartagena de indias, Colombian Caribean. Harmful Algae News 53 (9).

SHAH, M.; RAHMAM, M.; SO-JUNG, A.; JOON-BAEK, L. 2013. Presence of benthic dinoflagellates around coastal waters of Jeju Island including newly recorded species. Journal of Ecology and Environment 36 (4): $347-370$.

SHEARS, N.; ROSS, P. 2009. Blooms of benthic dinoflagellates of the genus Ostreopsis; an increasing and ecologically important phenomenon on temperate reefs in New Zealand and worldwide. Harmful Algae 8 (6): 916-925. 
TAYLOR, F. 1978. Dinoflagellates. Identification problems. Some specific preparations. Págs 143-147 en: Sournia, A. (ed.), Phytoplankton manual. Monographs on Oceanographic Methodology 6. United Nations Educational, Scientific and Cultural Organization. París.

TAYLOR, F.; HOPPENRATH, M.; SALDARRIAGA, J. 2008. Dinoflagellate diversity and distribution. Biodiversity and Conservation 17 (2): 407-418.

TAYLOR, W. 1960. Marine Algae of the Eastern Tropical and Subtropical Coasts of the Americas. University of Michigan Press. Ann Arbor. USA.

VALERIO, L.; DÍAZ, J. 2008. Distribución de dinoflagelados epifitos potencialmente tóxicos asociados a praderas de Thalassia testudinum en la isla la Tortuga, la Bahía de Mochima y Golfo de Cariaco, Venezuela. Boletín del Instituto Oceanográfico de Venezuela 47 (1): 47-58.

VEGA-SEQUEDA, J.; RODRÍGUEZ-RAMÍREZ, A.; REYES-NIVIA, C.; NAVAS-CAMACHO, R. 2008. Formaciones coralinas del área de Santa Marta: estado y patrones de distribución espacial de la comunidad bentónica. Boletín de Investigaciones Marinas y Costeras-INVEMAR 37 (2): 87-105.

VILA, M.; GARCÉS, E.; MASÓ, M. 2001. Potentially toxic epiphytic dinoflagellate assemblages on macroalgae in the NW Mediterranean. Aquatic Microbial Ecology 26 (1): 51-60.

YASUMOTO, T.; INOUE, A.; BAGNIS, R.; CARCON, M. 1979. Ecological survey on a dinoflagellate possibly responsible for the induction of ciguatera. Bulletin of the Japanese Society of Scientific Fisheries 45 (3). $395-399$. 